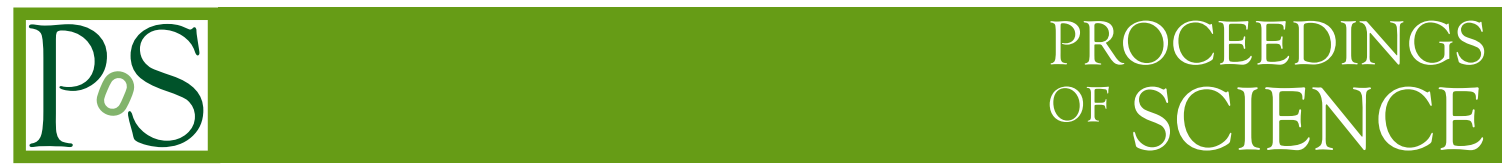

\title{
On Drinfel'd twists and their use in non-commutative geometry
}

\author{
Pierre Bieliavsky* \\ Université Catholique de louvain \\ E-mail: pierre.bieliavsky@gmail.com
}

\begin{abstract}
I will present some recent developments in the theory of non-formal Drinfel's twists. A Drinfel'd twist consists in a tool that allows to deform in an associative way any associative algebra that possesses a given symmetry. For instance the Moyal twist is a Drinfel'd twist for the abelian symmetry $\mathbb{R}^{d}$. I will report a general method for constructing Drinfel'd twists based on generally non-abelian symmetries. I will conclude by mentioning applications in various domains.
\end{abstract}

Frontiers of Fundamental Physics 14 - FFP14,

15-18 July 2014

Aix Marseille University (AMU) Saint-Charles Campus, Marseille

${ }^{*}$ Speaker. 